\title{
Research on Household Waste Detection System Based on Deep Learning
}

\author{
Li Mingyang ${ }^{1}$, Li Chengrong ${ }^{1 *}$ \\ ${ }^{1}$ Institute of Automation, Chinese Academy of Sciences, Intelligent Manufacturing Technology and System Research Center, Beijing \\ 100190
}

\begin{abstract}
Household waste is threatening the urban environment increasingly day by day for people's material needs increasing with the acceleration of urbanization. In this paper, a new waste sorting model is proposed to solve the problems of waste sorting. The style transfer was used to increase the data set to make some objects be sorted well. Then the rotational attention mechanism model was used to increase the accuracy of waste sorting of the blocked objects. The representation vector extraction module in the target tracking algorithm Deep Sort was replaced with Siamese network to make the network more lightweight. As a result, this paper effectively solves the current waste sorting tasks.
\end{abstract}

thus saving human resources.

\section{Introduction}

In recent years, with the rapid development of the world economy and the accelerating process of urbanization, people's material needs also increase. As a result, household waste is threatening the urban environment increasingly day by day. Waste is a misplaced resource, and reasonable classification and treatment of waste are extremely urgent [1]. Therefore, intelligent waste classification is very important for intelligent waste sorting and improving the efficiency of waste sorting.

The main process of waste sorting at present is as follows. In the mixed waste assembly line, when there is a waste target object passes, a color camera is used to take pictures. The image processing algorithm is used to identify the material type and position of the object to be captured in the picture. Then the information is sent to the downstream robot; and according to the received information, the robot grabs the waste target object and places it in the specified material frame, to realize automatic and intelligent waste sorting [2]. Image recognition technology is the core and most difficult link in waste sorting equipment. In the actual mixed waste disposal site, the phenomenon of dirt, damage, adhesion, and blocking between objects is very serious, so it is difficult to identify the recyclable waste effectively by the traditional image processing algorithm.

In recent years, deep learning has been continuously developed, and its theoretical achievements have been widely applied to many different life scenarios [3], such as image classification, target tracking, face recognition, and recommendation system. Based on the deep learning model, waste classification and detection tasks under dense waste scenarios can be effectively completed, and mechanized and efficient waste sorting can be realized,

\section{Related to This Paper}

To realize the goal of waste sorting, such as mechanization and high efficiency, and saving human resources, this paper focuses on the following problems. (1) Determine the waste classification and detection tasks. Use opensource Haihua waste data detection data sets and two different network models, namely, single-phase target detection YOLO v3 and CenterNet, for contrast experiment on the Haihua data set. (2) Collect certain types of MSW data, build waste detection data set locally, and put forward an almost no-parameter attention mechanism module in the feature extraction network of CenterNet to improve detection accuracy without affecting the detection speed. (3) Reduce the influence of blocking and illumination change on the accuracy of model detection in the process of target waste detection.

\section{Research on Innovation Points}

In the research process, three innovation points were mainly used to research with the help of a lot of literature which was applied to the waste sorting task. The three points are as follows:

1) To expand the data set and make the data set scenarios closer to the real scenarios, the style transfer algorithm was used; to solve the illumination change and blocking in the data, data enhancement was used; to deal with the inaccurate detection caused by blocking, the repulsive loss was applied.

2) The rotational attention mechanism module is introduced, and the three-branch structure is used to capture cross-dimensional interaction to calculate the

*Corresponding author's e-mail: limingyang2018@ia.ac.cn 
weight of attention. This attention module almost does not increase the calculation cost, and can effectively improve the waste detection accuracy without affecting the detection accuracy.

3) The representation vector extraction module in the target tracking algorithm Deep Sort is replaced with Siamese network to make the network more lightweight.

\subsection{Local Dataset Construction}

4,508 waste images were annotated locally, of which 4,012 were used as the training set and 496 as the verification set. According to the material, they were divided into seven categories, respectively, can, PP (polypropylene), PET (polyethylene terephthalate), HDPE (high-density polyethylene), paper, glass, and Tetra Pak packaging. The label corresponds to the category of each waste in each image and the position of the bounding box (top-left point and bottom-right point coordinates), as shown in Figure 1.

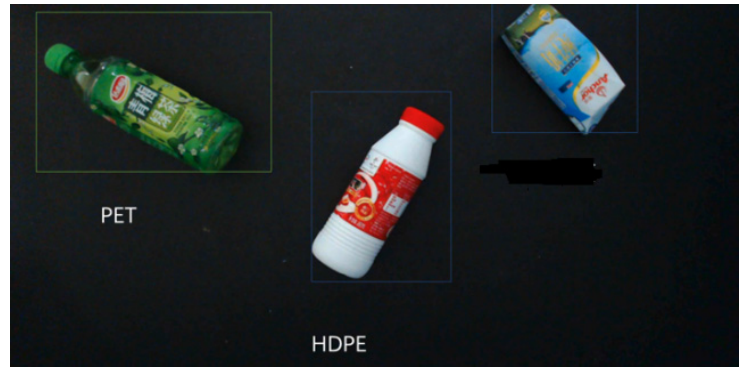

Fig1. Sample Local Data Annotation

\subsection{Style Transfer}

The style transfer algorithm is used to generate the source domain data of different textures. Each source domain image is used to remove the composite texture and get a random texture. The training on the data with different textures can guide the model to learn the texture invariant representation and pay more attention to the contour shape features of the model. Due to the large scale and high resolution of synthesized data, the style transfer algorithm needs to be efficient. Finally, the style transfer algorithm can lead to a variety of styles, which can transfer different image styles. Considering the above conditions, the Styleswap algorithm is finally adopted. Style-Swap [4] is a local matching algorithm based on image content structure and image texture structure. The network structure of the texture style transfer algorithm is shown in Figure 2.

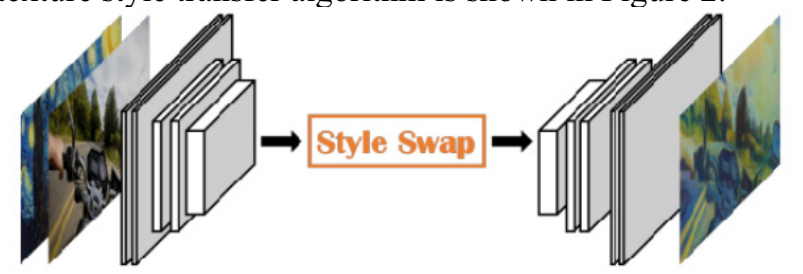

Fig2. Style Transfer Network Structure

As shown in Figure 3, through this algorithm, the texture of the style image can be used to synthesize the final style transfer image while retaining the contour shape of the original image.
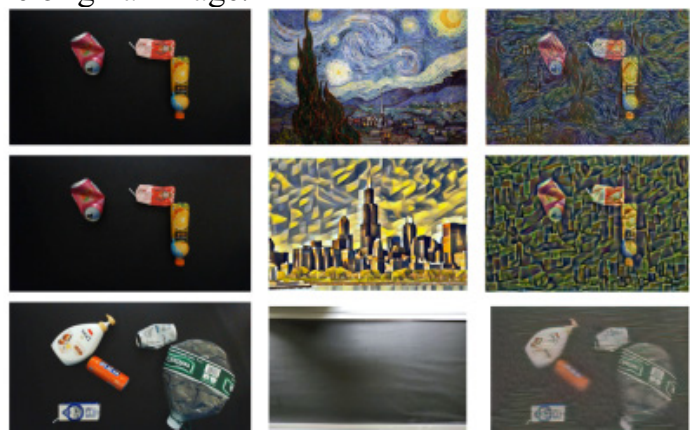

Fig3. Style Transfer Results

\section{Experimental Process}

\subsection{Haihua Waste Detection Data Set}

There are 205 categories of Haihua waste detection data sets. The data set is divided into two kinds. One is a singleclass data set, with a total of 80,000 waste images, and each image has only one category and provides the border information of the unique object in the image. The other is a multi-class data set, which contains 2,998 waste images in the training set and 1,000 in the verification set. Each image contains at most 20 categories, as shown in Figure 4. Data features: the category imbalance exists in multiclass data sets.
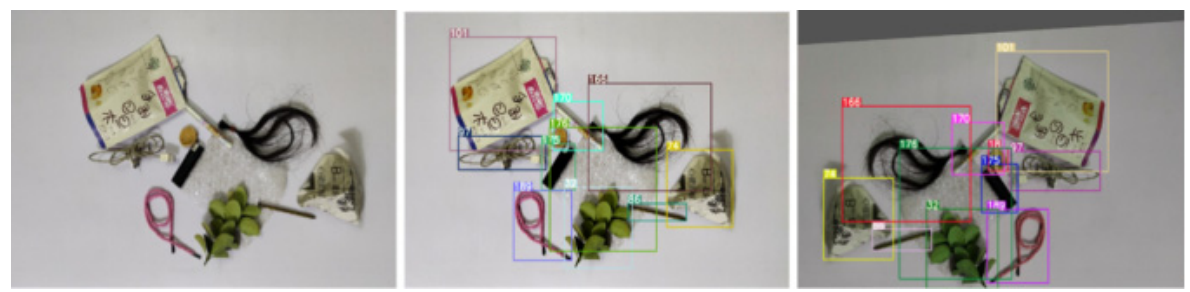

Fig. 4 Sample Data of Haihua

\subsection{End-to-end Target Detection Model}

The YOLO V3 model is the third iteration version of the YOLO [5] series algorithm. Compared with the previous two models, YOLOV3 designed Darkent-53, a basic network with stronger feature extraction capability. By adding the structure of Feature Pyramid [6], the capability of multi-scale detection of the model was improved, and YOLOV3 realized multi-class classification so that the model could adapt to complex tasks. The structure of YOLOV3 is shown in Figure 5. 


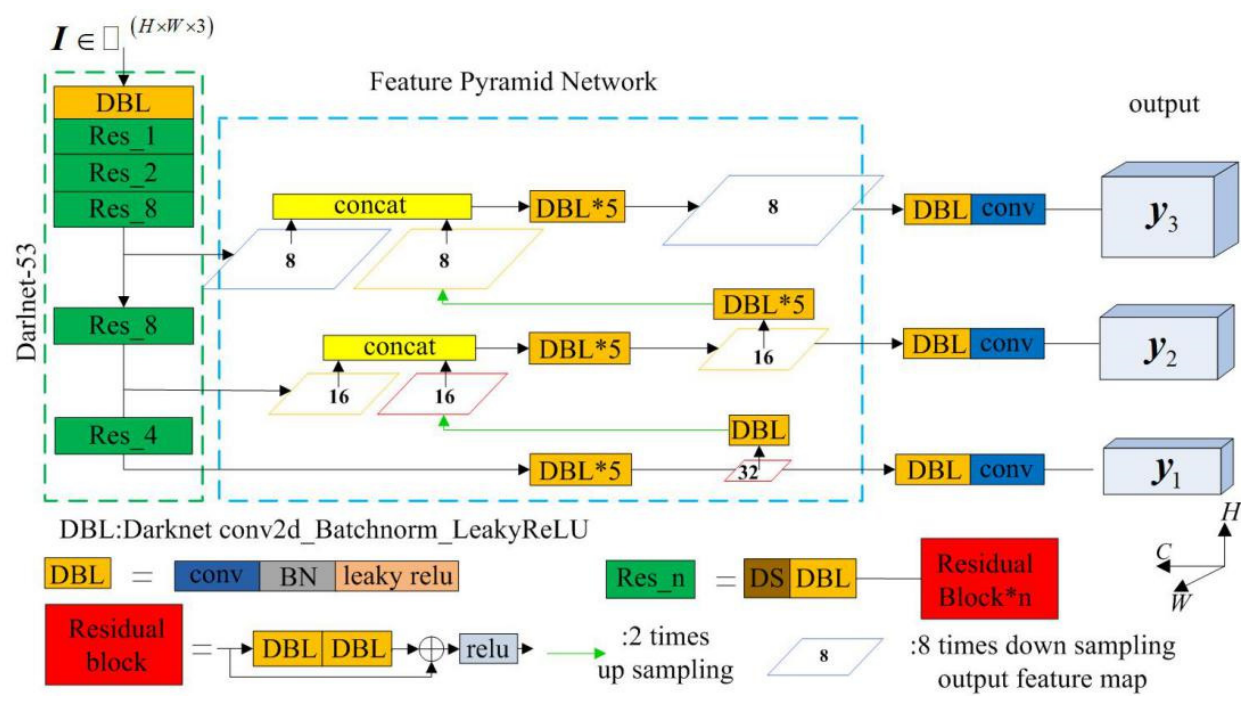

Fig. 5 YOLO V3 Structure

\subsection{Attention Mechanism}

In the human visual system, usually, the corner of the human eyes is used to deal with unimportant areas and more attention is allocated to important information to better establish visual structure. [7] The simulation of the human visual attention mechanism can be used to inhibit the invalid information in the network, strengthen the important information, which is good for the target detection task. The rotational attention module is put forward in this paper. For the tensor for input, the rotational attention module [8] uses a three-branch structure to capture cross-dimension interactions to calculate weight attention. The attention module almost does not increase the computational overhead and emphasizes the importance of multidimensional interaction instead of reducing the dimension [9], thus eliminating indirect corresponding between the channel and weight [10]. The specific structure of rotational attention is shown in Figure 6.

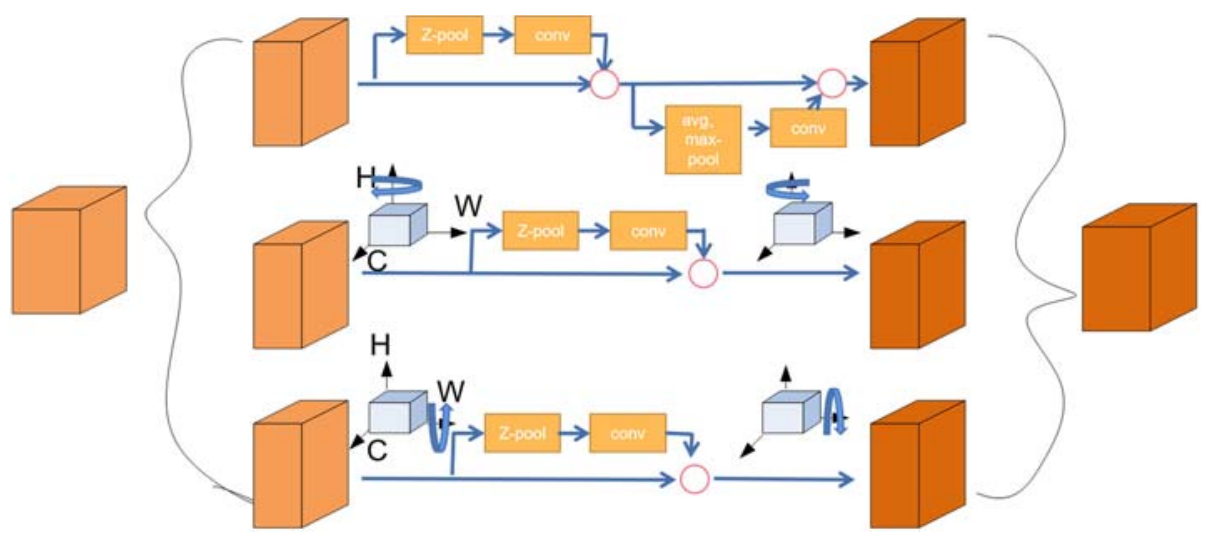

Fig. 6 Rotational Attention Module

\section{Comparative experiment and model effect}

The waste detection model is based on CenterNet [11] and
YOLO V3 [12], and the image size is $960 * 540$. Different Backbones were used to introduce the rotational attention mechanism module, and a comparative experiment was carried out on Haihua waste detection data set. The experimental results are shown in Table 1.

Table 1 Comparison of Experimental Results

\begin{tabular}{|l|l|l|l|}
\hline Backbone & Detectors & mAP & fps \\
\hline Resnet-50 & Yolo v3 & 0.873 & 31 \\
\hline Resnet-50+CBAM & Yolo v3 & 0.876 & 29 \\
\hline Resnet-50+RAM & Yolo v3 & 0.881 & 28 \\
\hline Resnet-50 & CenterNet & 0.880 & 33 \\
\hline
\end{tabular}




\begin{tabular}{|l|l|l|l|}
\hline Resnet-50+CBAM & CenterNet & 0.882 & 31 \\
\hline Resnet-50+RAM & CenterNet & 0.886 & 30 \\
\hline
\end{tabular}

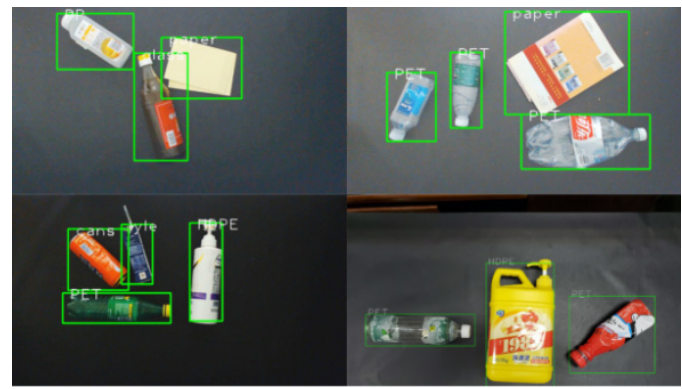

Fig. 7 Test Results

RAM+CenterNet was used to train on the locally annotated waste data set, and the average precision mAP on the test set reached $97.8 \%$, and the detection speed was 31.7 FPS. The detection effect is shown in Figure 7.

\section{Conclusion}

In this paper, a new waste sorting model was proposed to solve the problems of waste sorting. The style transfer was used to increase the data set so that some blocked objects can be sorted well. Then the rotational attention mechanism model was used to increase the accuracy of waste sorting. The representation vector extraction module in the target tracking algorithm Deep Sort was replaced with Siamese network to make the network more lightweight. As a result, this paper effectively solves the current waste sorting tasks.

\section{References}

1. Wei Xiaoxiao, Wang Xiaoming, Li Lei, et al. Temporal and spatial characteristics of municipal solid waste generation and treatment in China from 1979 to 2016 [J]. China Environmental Science, 2018, 38(10): 38333843.

2. Du Huanzheng, Liu Feiren. Difficulties in China's Municipal Domestic Waste Classified Collection and Solutions [J]. Journal of Xinjiang Normal University (Edition of Philosophy and Social Sciences), 2020, 41(01): 134-144+2.

3. X. Du, S. Tang, Z. Lu, J. Wet, K. Gai and P. C. K. Hung, "A Novel Data Placement Strategy for DataSharing Scientific Workflows in Heterogeneous Edge-Cloud Computing Environments," 2020 IEEE International Conference on Web Services (ICWS), Beijing, China, 2020, pp. 498-507.

4. Serranti, S., Bonifazi, G., Pohl, R., 2006. Spectral cullet classification in the mid infrared field for ceramic glass contaminants detection. Waste Manage. Res. 24(1), 48-59.

5. Redmon J, Divvala S, Girshick R, et al. You only look once: unified, real- time object detection[C]//IEEE Conference on Computer Vision and Pattern Recognition, 2016: 779-788.
6. Ojala, Timo, Pietikainen, et al. Multiresolution GrayScale and Rotation Invariant Texture and Classification with Local Binary Patterns [J]. IEEE Transactions on Pattern Analysis \& Machine Intelligence, 2002.

7. Dalal N, Triggs B. Histograms of oriented gradients for human detection [C] //Proceedings of 2005 IEEE Computer Society Conference on Computer Vision and Pattern Recognition. San Diego, CA, USA: IEEE， 2005: 886-893. [DOI: 10.1109 /CVPR. 2005. 177]

8. Bay H, Tuytelaars T, Gool L V SURF: Speeded up robust features[C]// Proceedings of the 9th European conference on Computer Vision - Volume Part I. Springer-Verlag, 2006.

9. Paulraj S G, Hait S, Thakur A. Automated Municipal Solid Waste Sorting for Recycling Using a Mobile Manipulator[C]// ASME 2016 International Design Engineering Technical Conference (IDETC). 2016.

10. X. Du, J. Xu, W. Cai, C. Zhu and Y. Chen, "OPRC: An Online Personalized Reputation Calculation Model in Service-Oriented Computing Environments," in IEEE Access, vol. 7, pp. 8776087768, 2019.

11. Ren S, He K, Girshick R, et al. Faster R-CNN: towards real- time object detection with region proposal networks[J].IEEE Transactions on Pattern Analysis \& Machine Intelligence, 2017, 39(6):11371149

12. Redmon J, Farhadi A. YOLO9000: better, faster, stronger [C]//EEE Conference on Computer Vision and Pattern Recognition, 2017: 6517-6525. 